\title{
Labyrinthe
}

$10 \mid 2001$

Numéro 10

\section{Famille et espace dans le Conte du Graal et ses trois dernières Continuations en vers (1170-1230)}

\section{Sébastien Douchet}

\section{(2) OpenEdition \\ 12 Journals}

Édition électronique

URL : http://journals.openedition.org/labyrinthe/1205

DOI : $10.4000 /$ labyrinthe.1205

ISSN : 1950-6031

Éditeur

Hermann

\section{Édition imprimée}

Date de publication : 1 octobre 2001

Pagination : 53-64

Référence électronique

Sébastien Douchet, «Famille et espace dans le Conte du Graal et ses trois dernières Continuations en vers (1170-1230) », Labyrinthe [En ligne], 10 | 2001, mis en ligne le 10 avril 2006, consulté le 01 mai 2019. URL : http://journals.openedition.org/labyrinthe/1205; DOI : 10.4000/labyrinthe.1205

Ce document a été généré automatiquement le 1 mai 2019.

Propriété intellectuelle 


\title{
Famille et espace dans le Conte du Graal et ses trois dernières Continuations en vers (1170-1230)
}

\author{
Sébastien Douchet
}

1 Partant du constat que les liens de parenté entretiennent avec l'espace des liens étroits, il nous est apparu que dans la matière du Graal en vers il est possible d'établir une typologie des lieux en fonction du rôle qu'ils jouent dans la constitution de l'identité familiale du héros Perceval ${ }^{*_{1}}$. Selon les lieux, mais aussi les auteurs, l'espace dans le texte tient un discours implicite sur les liens de parenté, la sexualité et la perpétuation du lignage. Le Conte $d u$ Graal et ses Continuations en vers ${ }^{2}$ nous semblent pouvoir se prêter à une telle analyse car le héros de Chrétien de Troyes, un orphelin de père qui part en quête du Graal, rencontre au cours de son errance certains de ses parents qu'il ne connaissait pas : sa cousine, mais aussi ses oncles, l'ermite et le Roi Pêcheur, des tantes, etc. Se déplacer à l'aventure dans le monde permet donc au héros de reconstituer son lignage et de se réapproprier une identité généalogique jusqu'alors inconnue. Toutefois, la mission du héros, Perceval, consiste avant tout à percer les mystères du Graal, comme le suggère son nom. En quoi la reconstitution des liens de famille participe-t-elle à cette mission? L'étude de quelques cas précis permet de comprendre la logique qui préside au départ en aventure, mais aussi la signification des lieux familiaux et le rôle qu'ils remplissent dans la constitution de l'identité héroïque. Il nous a semblé que mettre en scène et en espace la famille de Perceval était un moyen de souligner les qualités indispensables au héros pour mener à bien cette mission. Le roman construit et déconstruit ainsi des lieux et des liens de famille, tandis que leurs auteurs élaborent symétriquement leur texte comme le moyen de s'insérer dans une lignée littéraire, ou du moins narrative, démarche dont nous verrons la portée dans l'histoire des lettres.

2 Le cycle en vers de Perceval est encadré par deux lieux familiaux, féminins et masculins, qui font passer le héros de l'état d'enfant ou d'adolescent à celui de chevalier accompli et de seigneur de la terre du Graal. 
3 En amont, le Conte du Graal de Chrétien de Troyes s'ouvre sur le manoir de la mère, dans la Gaste Forêt. C'est ici que Perceval, le jeune nice, c'est-à-dire le naïf, l'innocent, a été élevé loin des tentations de la chevalerie : le père de Perceval et ses deux frères ayant été tués au combat, la mère a volontairement isolé son dernier fils pour lui éviter la même fin tragique. Ce manoir est l'espace de la protection maternelle, de la candeur. Entouré par la forêt, la vie y est rythmée par les jeux de chasse quotidiens du jeune homme, et le roman s'ouvre sur le renouveau du printemps et laisse l'impression d'un lieu suspendu dans une temporalité idyllique :

Einsi an la forest s'an antre / Et maintenant li cuers del vantre / Por le douz tans li resjoi / Et por le chant que il oï / Des oisiaus qui joie feisoient : / Totes cez choses li pleisoient ${ }^{3}$.

4 En aval, la Troisième Continuation se clôt sur le château du Roi Pêcheur, l'oncle de Perceval. Ce dernier en devient le seigneur après avoir réussi sa quête du Graal : il rend ainsi sa fertilité à la terre dévastée du Roi Pêcheur. Les réjouissances réunissent Arthur et tous les chevaliers de la Table Ronde autour d'un banquet approvisionné un mois durant par la grâce du Graal qui, tel une nouvelle corne d'abondance, sert à foison aux convives les mets les plus raffinés.

D'un lieu familial à l'autre Perceval quitte la maternité protectrice pour devenir lui-même une figure de roi paternel et nourricier. Il accomplit son initiation et de nice il devient le meilleur et le plus vertueux chevalier du monde, il s'insère dans sa famille et sa lignée en accomplissant son destin d'élu du Graal. Si bien qu'au moment de sa mort il monte au Paradis où il est placé à la droite de Dieu le Père, bienheureux parmi les bienheureux, intégré à une autre lignée, spirituelle et divine cette fois.

6 Trois lieux, manoir, château du Graal et Paradis circonscrivent donc les aventures de Perceval, dont ils sont les lieux originels et finaux. Ces lieux orientant tout le cycle romanesque vers une fin lui confèrent une visée doublement téléologique : téléologie mondaine en ce qui concerne l'accession à la royauté du Graal, téléologie eschatologique en ce qui concerne l'accession au Paradis.

7 Mais qu'en est-il du manoir maternel ? Pourquoi faut-il quitter ce lieu et débuter le roman par une rupture et un malheur, puisque le départ de Perceval, rappelons-le, fait mourir sa mère de chagrin? Nous savons que la mère, redoutant que son fils ne veuille lui aussi devenir chevalier, tente de le maintenir hors du monde. Le manoir permet donc d'entretenir Perceval dans un état de niceté originel, comme si la mère étouffait chez son fils toute forme de désir, en particulier celui de devenir chevalier comme son père et ses frères. Si le manoir est le lieu où étymologiquement l'on demeure (manere), en ce qui concerne Perceval, il est le lieu où l'on demeure dans l'absence de désir, dans l'incomplétude de son être et de son identité. La meilleure preuve en est qu'au début du roman Perceval ne connaît pas même son vrai nom, et croit qu'il s'appelle " mon fils ", puisque c'est ainsi que l'appelle sa mère... Son identité est donc soigneusement et volontairement placée dans l'ombre par la parole maternelle.

8 L'événement décisif qui pousse Perceval à quitter sa mère est sa rencontre avec des chevaliers qui, littéralement, l'éblouissent: c'est le reflet du soleil sur les armures rutilantes qui éveille en lui le désir de devenir leur égal.

Mes quant il les vit an apert / Que del bois furent decovert, / Et vit les haubers fremianz / Et les hiaumes clers et luisanz / Et les lances et les escuz / Que onques mes n'avoit veüz, / Et vit le vert et le vermoil / Reluire contre le soloil / Et l'or et l'azur et l'arjant / Si li fut mout et bel et gent / - Mes vos estes plus biaus que Deus. / Car fusse je ore autreteus, / Einsi luisanz et einsifez ! 
9 Au contraire de la parole maternelle, parole de l'ombre, le discours des chevaliers illumine la conscience de Perceval qui comprend alors qu'il désire plus que tout au monde porter les armes. Perceval semble donc entrer en aventures sous le coup d'une sorte d'atavisme chevaleresque qui le pousse à reproduire la vocation de son père et de ses frères : il réintègre ainsi déjà un peu sa lignée familiale, pour l'heure à son insu, et deviendra lui-même, au terme de son accession à la royauté du Graal, la figure paternelle déjà mentionnée. Il peut donc sembler schématiquement que quitter le lieu maternel permet d'accomplir son destin de chevalier pour accéder au statut de père. Toutefois, les Continuations ne laissent pas de poser problème dans la manière dont elles ont décidé de poursuivre les aventures du jeune Gallois, et de réélaborer l'image des lieux familiaux.

Le motif des trois lieux, initiaux et finaux, est repris au tout début de la Quatrième Continuation de Gerbert de Montreuil, ce qui confirme leur rôle central dans le cycle de Perceval.

11 Au début de ce roman, Perceval se réveille sous un arbre et le château du Graal, où il se trouvait la veille au soir, a disparu. Alors le narrateur fait dire à Perceval qu'il lui faut retourner au manoir de sa mère, sans même s'expliquer sur ce qui motive cet acte. Le héros monte donc à cheval et, peu de temps après, arrive devant une muraille circulaire. Il frappe à la porte. Un vieillard vêtu de blanc lui ouvre les portes de ce lieu, de l'intérieur duquel s'élèvent des rires et des chansons de joie. Pour le narrateur, cette fois explicite, il s'agit du paradis sur terre. Nous retrouvons bien les trois lieux dont nous parlions auparavant, château, manoir et Paradis. Perceval voudrait accéder au Paradis, mais le vieillard lui refuse l'entrée à cause de l'état peccamineux dans lequel il se trouve encore. Perceval ne s'est pas encore amendé de tous les péchés qu'il a commis au cours de sa vie et c'est pourquoi il est également expulsé du château du Roi Pêcheur, n'étant pas encore assez pur pour percer les mystères du Graal. Il se rend alors chez sa mère. Le début de la Quatrième Continuation fait donc accomplir à Perceval un mouvement de retour en arrière qui apparaît comme une régression dans son cheminement spatial. De plus, il met implicitement en relation le manque de vertu du héros et le retour chez la mère. La suite du récit montre en effet que le manoir maternel est un lieu potentiellement dangereux, qui met en scène la tentation de l'inceste.

Alors qu'il n'en avait pas été fait mention chez Chrétien de Troyes, Perceval retrouve dans le manoir une sœur, déjà inventée cependant par la Deuxième Continuation de Wauchier de Denain. Dans la Quatrième Continuation, il l'emmène au Château des Pucelles et la confie à ses habitantes : cent vierges placées sous la tutelle de la tante de Perceval. Après cela, il ne sera plus question du manoir maternel: c'est une question liquidée, comme s'il s'agissait d'un lieu problématique du roman. Ce qu'il y a d'étrange dans cet épisode est cette nécessité qu'ont éprouvée les continuateurs de faire revenir Perceval au manoir, mais aussi de peupler ce lieu premier et originel d'un nouveau membre de la famille, la sœur, alors que chez Chrétien de Troyes le manoir restait vide après la mort de la mère au début du Conte du Graal. Le manoir est réinvesti d'une figure familiale, mais pour mieux le vider à nouveau! Il y a donc autour de ce lieu qu'est le manoir une configuration qui se répète et qu'il faut interroger. Dans la Deuxième Continuation, lorsque Perceval arrive au manoir de sa mère et reconnaît sa sœur, l'on assiste à une scène d'effusion qui donne lieu à des commentaires sous cape des domestiques. N'ayant pas reconnu Perceval, ceux-ci croient que la sœur se livre à un inconnu :

Atant li sergent venu sont, / Qui la table metre voloient ; / Molt se mervoillent quant il voient

/ Que lor demoiselle besoit / Li homs qui estranges estoit : / Antr'aus lou tenoient a mal ${ }^{5}$. 
Le trouble de cette situation d'allure incestueuse est d'autant plus grand que la description qui est donnée de la sœur la rapproche étrangement de Blanchefleur, l'amie « officielle » de Perceval :

Fors d'une belle chambre vint / Une molt très belle pucelle, / Blanche con fleur de lis novelle ${ }^{6}$

Le doute est levé sitôt que la jeune fille révèle aux serviteurs qu'il s'agit de son frère. Mais cet épisode donne sens à l'invention du personnage de la sœur : le manoir, lieu premier, est le lieu d'un interdit, du tabou de l'inceste et de l'impossible réalisation du désir amoureux. La constitution de l'image de la fraternité passe par le soupçon de l'anormalité du désir. Par ailleurs, lorsque Perceval revient sur le lieu idéal de son enfance, il se recueille également sur la tombe de sa mère, tombe qui ne se trouve pas sur les lieux du manoir, mais un peu plus loin dans la forêt, dans la chapelle de son oncle ermite.

En plaçant le personnage de la sœur dans le château des pucelles et en éloignant du manoir la dépouille mortelle de la mère, le texte souligne fortement la nécessité qu'il y a de vider le lieu premier de la parentèle de Perceval : la présence de la sœur aussi bien que de la mère mettait en évidence un obstacle symbolique à la réalisation des désirs de Perceval. Vider le lieu originel qu'est le manoir, c'est donc effacer le risque de l'inceste, mettre en scène et en fiction sa prohibition. Ce faisant, le roman fait littéralement disparaître les membres féminins de la famille Perceval, les place hors de son regard et fait ainsi disparaître, tout en l'ayant convoqué, le risque aberrant de l'adultère, péril pour le lignage et la famille à venir.

Mais la question du désir amoureux «normal » est envisagée de manière différenciée par les trois Continuations, qui ont chacune une coloration «idéologique » qui leur est propre. Autant la Deuxième Continuation fait une large place aux aventures courtoises, autant la Troisième Continuation et la Quatrième Continuation font preuve d'une grande défiance à l'égard du désir amoureux. Cette inflexion vers une sorte rigorisme en matière de sexualité peut être abordée à partir d'un prologue en vers rédigé par un auteur anonyme et ajouté postérieurement au Conte du Graal : le Bliocadran.

Dans ce texte des détails nouveaux sont inventés sur la famille paternelle du héros et notamment celui-ci : le père de Perceval a été élu parmi ses frères pour donner naissance au héros qui accomplira la quête du Graal, pour cette raison qu'il est le plus pur et le plus vertueux de sa fratrie. De la même manière, la question de la chasteté du héros est importante dans la Troisième Continuation et la Quatrième Continuation, et les apparitions de prêtres dans la fiction sont nombreuses pour rappeler Perceval à son devoir en la matière. Pour réussir cette quête, le roman s'enferre alors dans un paradoxe: Perceval doit incarner le point d'aboutissement de la pureté de sa lignée, en même temps que cette pureté condamne cette même lignée. Perceval en est en effet le seul descendant mâle, et devant rester chaste il se prive de descendance. La quête échue à sa famille aboutit donc à une disparition de la lignée, condition nécessaire au succès de la mission du Graal.

Cette évolution vers un discours beaucoup plus clérical et moralisateur, chez Manessier et Gerbert de Montreuil, va de pair avec une explication de plus en plus christianisée des mystères du Graal : alors que chez Chrétien de Troyes le Graal ne fait que fournir au Riche Roi une nourriture spirituelle, sous la forme d'une hostie, l'influence de la matière en prose du cycle du Graal, et notamment de Robert de Boron, fait du Graal, le récipient qui a recueilli le sang du Christ. L'on apprend ainsi dans la Troisième Continuation que le Graal a 
été rapporté en Occident par la famille de Perceval, et notamment par sa mère : la famille maternelle du héros est donc celle par qui a eu lieu la translatio des reliques du Christ.

Ce premier mouvement dans l'espace, accompli grâce à la famille de Perceval, est un horsscène, et le cycle du Graal fera advenir une autre translatio, qui n'aura lieu que dans la Troisième Continuation : il s'agit de l'assomption du Graal et de Perceval, qui sont accueillis dans le sein du Père. L'errance de Perceval dans un espace « horizontal » et mondain est donc in fine compensée par ce mouvement vertical et épiphanique. Dans l'esprit des deux dernières Continuations, l'errance du chevalier dans le monde ne vise donc pas, en dernière instance, à reconstituer les membra disjecta de sa famille, dont il ignore tout au moment de son départ en aventure. Cette reconstitution n'est plus que le pré-requis à une sanctification du héros, dont on découvre peu à peu qu'il appartient à une famille d'élus dès le temps du Christ. L'orientation donnée au cycle par les Continuations est donc délibérément didactique et édifiante : la vraie place de Perceval se trouve dans le sein de Dieu, sa vraie lignée est spirituelle, et ses aventures s'inscrivent dans la continuité des récits du Nouveau Testament, eux-mêmes dans la continuité des récits de l'Ancien Testament . De plus, Manessier et Gerbert de Montreuil ne manquent jamais de glisser dans les discours de leurs personnages quelque sermon sur les sodomites ou la virginité. La meilleure illustration de ce glissement clérical réside dans le traitement des aventures courtoises de Perceval et Blanchefleur dans le Conte du Graal, qui, dans la Troisième Continuation, débouchent sur un mariage; mais celui-ci n'est pas consommé, de peur de contrevenir aux prescriptions de l'Église sur la fornication. À aucun moment il n'est question de la légitimation du lignage par le sang et/ou la terre en ce qui concerne Perceval : il refuse toutes les unions et tous les fiefs qui lui sont proposés en récompense de ses exploits lors de ses aventures mondaines. Le roman opère donc peu à peu un déplacement de la problématique féodale vers une problématique mystique : l'accession à la royauté mystique du Graal, seule garante de la constitution d'une lignée, fondée par une mission biblique, chrétienne et édifiante.

Le genre du roman d'errance constitue en lui-même un excellent moyen littéraire de parvenir à cette fin. L'errance est d'une part nécessaire à la production du texte - les aventures permettent de repousser la fin du roman - et à la mise à l'épreuve du héros, qui se doit de se montrer exemplaire; d'autre part elle est également nécessaire à la portée idéologique du récit, en ce que le non-attachement au lieu permet de laisser flotter le mystère sur l'identité du personnage. Cela souligne l'idée que le roman ne vise pas à perpétuer la lignée de Perceval dans le cadre d'une généalogie, fondée sur la possession de la terre, mais sur l'ascèse guerrière et le renoncement mondain. Enfin l'errance permet l'expérimentation du désir qui est en dernière instance refusé et dénié.

21 En outre cette problématique de la reproduction et de la perpétuation de la lignée, que l'on observe sur le plan du récit fictionnel, est reprise sur le plan métatextuel par la création et la constitution elles-mêmes du cycle de Perceval en vers.

Cette problématique est tout entière contenue dans le très célèbre prologue du Conte $d u$ Graal de Chrétien de Troyes. L'image du semeur-écrivain placée en tête du texte fondateur du mythe du Graal n'a pas échappé à ses continuateurs, notamment à Gerbert de Montreuil qui, à l'orée de son propre roman, la réemploie pour marquer son appartenance à la lignée des continuateurs du Conte. La question de la filiation littéraire est ici essentielle pour comprendre que les cycles des Continuations en vers légitiment leur discours par cette appartenance à une famille littéraire, qui a en partage un genre (le 
roman d'aventures), une matière (la matière percevalienne du Graal) et une forme (l'octosyllabe en langue vernaculaire).

Normalement légitimé dans un prologue et par le recours aux auctoritates, le jeune roman médiéval s'invente et se rattache à des sources externes et prestigieuses, afin de combler l'hiatus qui le sépare des origines latines de la langue vernaculaire. Or la Continuation n'a par définition pas de prologue. Gerbert de Montreuil invente donc à cette fin un autre procédé : il déplace son inscription dans une lignée littéraire du plan du discours au plan du récit, et fictionnalise, par une référence intertextuelle, sa filiation avec Chrétien de Troyes. En effet, dès le vers 348 de sa Continuation, Gerbert fait rencontrer à Perceval un paysan :

Perchevaus d'autre part regarde, / Vit un manoir dedens le lai, / Unques en roman ne en lai / N’oï parler de si plaisant. / Perchevaus voit un païsan / Qui blé senme en une couture, / Envers lui en vient a droiture?

Tout à fait inhabituelle dans le roman du Graal, la figure du paysan vaut ici en ce qu'elle fait écho aux célébrissimes vers d'ouverture du Conte du Graal :

Qui petit seme, petit quialt, / Et qui auques recoillir vialt, / An tel leu sa semance espande / Que fruit a cent dobles li rande; / Car an terre qui rien ne vaut / Bone semance i seche et faut. / Crestïens seme et fet semance / D'un romans que il ancomance / Et si le seme an si bon leu / Qu'il ne puet estre sanz grant preu ${ }^{8}$.

La métaphore de la semaille est intéressante à double titre: d'une part Chrétien de Troyes, qui ne place pas son œuvre sous une quelconque autorité littéraire, mais en fait le fruit de son propre labeur, se présente comme auteur, au sens propre (augere) de celui qui fait augmenter, croître. La production littéraire est donc envisagée comme une création, qui donne un surplus de sens. D'autre part ce prologue propose de l'activité littéraire une métaphore germinative, métaphore entérinée par l'inachèvement du roman, qui laisse ouverts tous les possibles littéraires.

Dès lors, la couture (culture, labour, labeur), par le jeu d'homonymie suggéré par Gerbert de Montreuil, se fait également suture, ravaudage, rhapsodie peut-être. La filiation littéraire volontairement soulignée par Gerbert de Montreuil inscrit sa Continuation dans un lignage fondé par les premières paroles de Chrétien de Troyes : les fruits de la semence répandue par Chrétien de Troyes dépassent son œuvre. Et si chaque auteur imprime sa marque personnelle à la matière du Graal, il est un dénominateur commun qui renforce l'unité des Continuations et justifie la continuité générique de l'une à l'autre comme de la graine au fruit : la forme versifiée en octosyllabes. D'un point de vue métatextuel, la collusion entre lien de parenté et espace est ainsi, nous semble-t-il, également pertinente. L'espace d'écriture en puissance qu'est la forme de l'octosyllabe souligne le lien entre Chrétien de Troyes et ses rejetons littéraires : car ce lien est le vers, perpétuel retour du même, dont l'achèvement est inachèvement en ce que la rime appelle l'écho, soit le retour du même sous une autre forme. Le vers est étymologiquement versura, moment où le paysan retourne son araire et forme les sillons, la «couture ». L'image de l'auteursemeur-laboureur qui déploie son texte dans le vers justifie aussi l'écriture inachevée et ouverte de la Continuation.

En ce sens, peut-être faut-il analyser la fin de la Troisième Continuation, décevante à bien des égards, comme l'épuisement de la matière percevalienne, de la glèbe qui ne peut plus rien rendre. Le roman, au tournant du XIII ${ }^{e}$ siècle, se tourne d'ailleurs vers la prose pour continuer à s'écrire. Plus aucun roman en vers de Perceval ne sera écrit dans l'histoire de la littérature française : comme la lignée de Perceval, la lignée des continuateurs en vers 
s'éteint. Ce qui se produit sur le plan de la fiction se reproduit sur le plan du discours et de l'énonciation. Un peu comme Gerbert de Montreuil a fait passer la figure du semeur du prologue, c'est-à-dire du discours de l'auteur, à la fiction, c'est-à-dire au récit.

$\mathrm{Au}$ total, la question de la lignée et de son rapport à l'espace renseigne sur la manière dont est envisagé un mythe littéraire en cours de constitution comme celui du Graal. Il permet d'en comprendre certaines modalités d'écriture, mais aussi les orientations idéologiques, principalement théologiques, qui les informent. Il reste encore beaucoup à dire sur cette somme romanesque, sur tous les lieux qu'elle évoque et les valeurs qui y sont attachées : faire commencer et finir ce cycle sur deux figures du manque familial, l'absence de père et l'absence de descendance, est selon nous l'indice que la jeune matière romanesque en langue vernaculaire s'interroge sur sa légitimité, et les conditions auxquelles sa perpétuation est possible. Mais dans le mouvement même où est posée cette interrogation, la Troisième Continuation achève le cycle qui s'est consumé dans une forme devenue désuète, et s'efface pour céder la place à une autre lignée de romans, voués à un brillant avenir : les romans en prose.

\section{BIBLIOGRAPHIE}

Chrétien de TRoyes, Le Conte du Graal (Perceval), publié par Félix Lecoy, 2 volumes d'après la copie de Guiot, manuscrit B.N.F. Fr. 794, Paris, Champion, 1972-1975.

WAUCHIER DE DENAIN, The Continuations of the Old French Perceval of Chrétien de Troyes, The Second Continuation, volume IV, publié par William Roach, Philadelphia, University of Pennsylvania Press et American Philosophical Society, 1971.

MANESSIER, The Continuations of the Old French Perceval of Chrétien de Troyes, The Third Continuation, volume V, publié par William Roach, Philadelphia, University of Pennsylvania Press et American Philosophical Society, 1983.

GERBERT DE MONTREUIL, La Continuation de Perceval, éd. Mary Williams, tomes I et II (vers 1-7020 et 7021-14078), Paris, Champion, 1922-1925.

Gerbert De MonTReuI, La Continuation de Perceval, éd. Marguerite Oswald, tome III, Paris, Champion, 1975.

The Text of the Bliocadran, éd. Albert Wilder Thompson, Romance Philology IX, 1955-1956, p. 205-209.

\section{NOTES}

1.* Cet article s'inspire de recherches de doctorat sur représentation de l'espace dans la matière du Graal en vers. Le sujet est déposé à l'université de Paris X-Nanterre, sous la direction de M. le Professeur D. Boutet. Son auteur est actuellement ATER à l'université Paul Valéry-Montpellier III.

2. Le corpus ici envisagé comporte le Conte du Graal, roman inachevé de Chrétien de Troyes (vers 1180), sa suite inachevée de Wauchier de Denain (Deuxième Continuation, vers 
1205-1210), la suite, achevée, de la Deuxième Continuation, de Manessier (Troisième Continuation, avant 1230), et l'œuvre de Gerbert de Montreuil, la Quatrième Continuation, qui, bien que postérieure, s'intercale entre la Deuxième Continuation et la Troisième Continuation (vers 1230). Nous n'envisageons pas ici le cas de la Première Continuation, qui ne prend pas Perceval pour héros principal.

3. Conte du Graal, v. 85-90 : Ainsi entra-t-il dans la forêt et aussitôt son cœur, au plus profond de lui-même, fut transporté de bonheur par la douceur du temps et le chant des oiseaux qui s'en donnaient à cœur joie. Tout cela le rendait heureux.

4. Voir Conte du Graal, v. 127-136;179-181 : Mais quand il les vit à découvert, sortant du bois, et qu'il vit les hauberts qui bruissaient et les heaumes clairs et brillants, et les lances et les boucliers qu'il n'avait jamais vus, quand il vit le vert et le vermeil reluire au soleil, et l'or et l'azur et l'argent, le spectacle lui parut extraordinaire de beauté et de grandeur. - Mais vous êtes plus beaux que Dieu. Ah! si je pouvais être comme vous, tout brillant et fait comme vous!

5. Voir Deuxième Continuation, v. 23704-23709 : À ce moment les serviteurs arrivèrent pour dresser la table. Ils furent stupéfaits de constater que leur maîtresse embrassait un homme étranger. Entre eux ils condamnèrent cette attitude.

6. Voir Deuxième Continuation, v. 23586-23600 : Sortant d'une chambre arriva une très belle jeune fille, blanche comme la fleur de lis qui vient d'éclore.

7. Voir Quatrième Continuation, v. 344-350 : Perceval regarda de l'autre côté et vit une maison au milieu du lac. Jamais dans un roman ou dans un lai l'on n'a entendu parler de lieu si agréable. Perceval vit alors un paysan qui semait son blé dans les labours. Il alla tout droit à lui.

8. Voir Conte du Graal, v. 1-10: Qui sème peu récolte peu, et qui veut faire une belle récolte doit planter sa semence en un lieu propre à la lui rendre au centuple. Car en terre qui ne vaut rien, la semence sèche et meurt. Chrétien plante et sème un roman qu'il commence, et il le sème en un si bon lieu qu'il ne pourra pas ne pas lui rapporter beaucoup.

\section{RÉSUMÉS}

Dans les romans en vers du Graal ( $\mathrm{XII}^{\mathrm{e}}$-XIII ${ }^{\mathrm{e}}$ siècle), espace et liens de familles entretiennent d'étroites relations. En effet, l'espace apparaît comme la catégorie de représentation la plus propre à construire l'identité familiale, à en exprimer les enjeux et les problématiques, et ce aussi bien sur le plan narratif que sur le plan littéraire. L'étude de figures clef telles que le manoir maternel, le château du Graal, le Paradis sur terre ou bien la semaille permet de l'illustrer.

"Family and space in the Conte du Graal and his three last Continuations in verses (1170-1230)". In the verse Grail romance ( $12^{\mathrm{e}}-13^{\mathrm{e}}$ centuries), space and family links are closely related. Thus, space seems to be the best category of representation able to build the family identity, to express its main knots and problems considering the narrative and literary history as well. This point is illustrated by a study of key-figures as the mother's home, the Grail castle, Paradise on earth or the sowing. 


\section{AUTEUR}

\section{SÉBASTIEN DOUCHET}

Sébastien Douchet, né en 1973, est ancien élève de l'ENS de Fontenay/Saint-Cloud et agrégé de Lettres Modernes. Il possède également un DEA d'histoire de l'art. ATER à l'université Paul Valéry-Montpellier III, il prépare actuellement une thèse sous la direction de M. le Professeur D. Boutet, sur le thème de la présentation de l'espace dans la matière du Graal en vers. Sebdouchet(at)free.fr 